\title{
Entanglement entropy of cosmological perturbations for S-brane Ekpyrosis
}

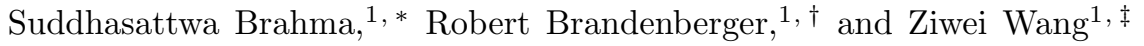 \\ ${ }^{1}$ Department of Physics, McGill University, Montréal, QC, H3A 2T8, Canada
}

(Dated: September 29, 2020)

\begin{abstract}
We calculate the entanglement entropy of scalar perturbations due to gravitational non-linearities present in any model of canonically-coupled, single-field ekpyrosis. Specifically, we focus on a recent model of improved ekpyrosis which is able to generate a scale-invariant power spectrum of curvature perturbations and gravitational waves as well as have a non-singular bounce due to an S-brane at the end of ekpyrotic contraction. By requiring that the entanglement entropy remians subdominant to the thermal entropy produced during reheating, we get an upper bound on the energy scale of the bounce.
\end{abstract}

\section{INTRODUCTION}

In a recent paper [1] we studied the momentum space entanglement entropy between sub- and super-Hubble modes generated by the intrinsic gravitational nonlinearities. We applied the formalism to inflationary cosmology and found that the entanglement entropy is a growing function of time since the phase space of super-Hubble modes is increasing. By demanding that the entanglement entropy remain smaller than the thermal entropy after reheating, we found an upper bound on the duration of inflation which is consistent with the constraint [2] coming from the trans-Planckian censorship conjecture [3. In this paper we will apply the formalism developed in [1] to the new version of the Ekpyrotic scenario recently proposed by two of us [4]. Once again, the phase space of super-Hubble modes is an increasing function of time, and hence the entanglement entropy will be increasing. By demanding that the entanglement entropy at the end of the phase of Ekpyrotic contraction be smaller than the thermal entropy after the bounce, we find an upper bound on the energy scale of the bounce.

The Ekpyrotic scenario [5] is a promising alternative to cosmological inflation. The scenario is based on the assumption that the cosmological scale factor $a(t)$ is decreasing very slowly

$$
a(t) \sim t^{p} \text { with } 0<p \ll 1
$$

as a function of physical time $t$, where $t<0$ in the contracting period. In the context of Einstein gravity, this time dependence of the scale factor can be obtained if matter is dominated by a scalar field with a negative exponential potential. Such potentials are ubiquitous in string theory compactifications (see e.g. [6]). A contracting universe with $w>1$ leads to a dilution of spatial curvature and of anisotropies [7]. It is also an attractor in initial space 8]. These are significant advantages compared to other cosmological models with a contracting phase (see e.g. 9] for a review of bounce scenarios).

\footnotetext{
*Electronic address: suddhasattwa.brahma@gmail.com

${ }^{\dagger}$ Electronic address: rhb@physics.mcgill.ca

${ }^{\ddagger}$ Electronic address:
}

Recently, two of us [4] proposed a new version of the Ekpyrotic scenario in which an S-brane (see e.g. [10]) arising at string densities mediates the non-singular transition between contraction and expansion. If the S-brane has zero shear, then roughly scale-invariant spectra of both cosmological perturbations and gravitational waves are generated [11], with two consistency relations between the four basic cosmological observables (the amplitudes and tilts of the scalar and tensor spectra). The decay of the S-brane after the bounce naturally generates [12] the radiation of the post-bounce universe. In this paper we will consider entropy constraints on this new scenario.

In the following section we briefly review the equations which describe the phase of Ekpyrotic contraction. In Section III we summarize the framework for computing momentum space entanglement between sub- and superHubble modes developed in [1]. We then turn to the computation of the entanglement entropy at the end of the phase of Ekpyrotic contraction, as a function of the Hubble parameter at that time, and derive the resulting upper bound on the energy scale of the bounce by demanding consistency with the second law of thermodynamics for the full time evolution of the model.

We close this section with some general remarks. We already introduced the cosmological scale factor $a(t)$ which yields the Hubble expansion rate

$$
H(t)=\frac{\dot{a}}{a}
$$

where an overdot represents a derivative with respect to time. The inverse of $H$ is the Hubble radius. The Hubble radius plays a crucial role concerning cosmological perturbations (see e.g. [13, 14 for reviews): on length scales smaller than the Hubble radius fluctuations oscillate. On super-Hubble scales the oscillations freeze out, the fluctuations can be squeezed and classicalize [15]. Both during an expanding inflationary cosmology and during Ekpyrotic scenario the Hubble radius decreases in comoving coordinates, and in both scenarios a natural assumption is that fluctuations start as quantum vacuum perturbations on sub-Hubble scales. The modes we have access to are the ones which have been able to classicalize. Hence, it is natural to divide the total Hilbert space of fluctuation modes into sub- and super-Hubble modes, and to 
study the entanglement entropy between these two sets of modes. Since the phase space of super-Hubble modes is increasing as a function of time, the resulting entanglement entropy will also be increasing. In order to be consistent with the entropy in the expanding radiation phase, the entanglement entropy at the end of the contracting phase cannot be too large. It is the resulting constraint on the model which we will here explore.

We will be using natural units in which the speed of light, Planck's constant and Boltzmann's constant are set to 1 . We denote the energy density by $\rho$ and the pressure by $P$, keeping the symbol $p$ for the exponent appearing in the expression for the time evolution of the scale factor (to be consistent with the usual notation in papers on the Ekpyrotic scenario).

\section{DESCRIPTION OF S-BRANE EKPYROSIS}

In the usual realizations of the Ekpyrotic scenario, the slow contraction phase is driven by a scalar field with negative exponential potential [5]

$$
S=\int d t d^{3} x\left(-\frac{1}{2} \partial^{\mu} \varphi \partial_{\mu} \varphi+V_{0} e^{-\sqrt{\frac{2}{p}} \frac{\varphi}{M_{\mathrm{P} 1}}}\right),
$$

where $M_{\mathrm{Pl}}$ is the Planck mass. Since the potential is steep, the field is not slowly rolling and we can find an approximate solution by ignoring the Hubble parameter $H$. This approximation is further justified since during Ekpyrosis the equation of state parameter $w \equiv \frac{P}{\rho} \gg 1$ and hence the contraction of the universe is very slow. Using this approximation, we find that the scalar field evolves according to

$$
\varphi(t)=\sqrt{2 p} M_{\mathrm{Pl}} \log \left(-\sqrt{\frac{V_{0}}{p}} \frac{t}{M_{\mathrm{Pl}}}\right) .
$$

Further, using the relation $\dot{\varphi}^{2}=-2 \dot{H} M_{\mathrm{Pl}}^{2}$, we find that

$$
\dot{H}=-\frac{p}{t^{2}}, \quad H=-\frac{p}{-t}, \quad a=a_{0}(-t)^{p} .
$$

One can check the self-consistency of our approximation by calculating the equation of state

$$
\begin{aligned}
w & =\frac{P}{\rho}=\frac{-\rho+\dot{\varphi}^{2}}{\rho} \\
& =\frac{-3 H^{2} \mathrm{~m}_{\mathrm{pl}}^{2}-2 \dot{H} \mathrm{~m}_{\mathrm{pl}}^{2}}{3 H^{2} \mathrm{~m}_{\mathrm{pl}}^{2}}=-1+\frac{2}{3 p} \gg 1 .
\end{aligned}
$$

From the above, the "slow-roll" parameters can be calculated in a straightforward manner. Note that only the first order slow-roll parameter is non-zero and is given by

$$
\epsilon=-\frac{\dot{H}}{H^{2}}=\frac{1}{p}, \quad \eta \equiv \frac{\dot{\epsilon}}{H \epsilon}=0 .
$$

It will be beneficial for us later on to rewrite the quantities above in terms of conformal time

$$
\tau(t)=\int \frac{d t}{a(t)}=-\frac{1}{1-p}(-t)^{1-p} .
$$

The background quantities are given by

$$
\begin{aligned}
a(\tau) & =\tilde{a}_{0}(-\tau)^{\frac{p}{1-p}} \\
H(\tau) & =p(1-p)^{-\frac{1}{1-p}}(-\tau)^{-\frac{1}{1-p}} \\
\mathcal{H}(\tau) & \equiv \frac{d a}{a d \tau}=-\left(\frac{p}{1-p}\right) \frac{1}{\tau} .
\end{aligned}
$$

In 4 it was suggested that an S-brane (a space-like brane) will be generated once the background energy density reaches the string scale. The S-brane is an object with vanishing energy density and negative pressure (since it has positive tension). Hence, it is an object which violates the NEC (null energy condition) and allows for the nonsingular transition between the Ekpyrotic contracting phase and a radiation dominated phase of expansion.

\section{STATES FOR SUPER-HUBBLE MODES}

In order to study the entanglement entropy due to mode-coupling between cosmological perturbations, we study linear fluctuations about a spatially flat FLRW metric. In longitudinal gauge, the metric can be written as [13, 14]

$$
\mathrm{d} s^{2}=-a^{2}(\tau)\left[\mathrm{d} \tau^{2}(1+2 \phi)-(1+2 \psi) \mathrm{d} \mathbf{x}^{2}\right],
$$

where $\phi$ and $\psi$ are the metric perturbation variables. A particularly useful variable is the curvature fluctuation $\zeta$ in uniform density gauge which is given by

$$
\zeta=-\psi+\frac{H}{\dot{\rho}} \delta \rho
$$

where $\rho$ is the background energy density and $\delta \rho$ is the density perturbation. If matter is a single field minimally coupled to Einstein gravity (as is the case in the Ekpyrotic scenario), the variable in terms of which the action for fluctuations has canonical form is

$$
v(\boldsymbol{x}, \tau) \equiv z(\tau) \zeta(\boldsymbol{x}, \tau)
$$

where

$$
z(\tau)=\sqrt{2 \epsilon} a M_{\mathrm{Pl}} c_{s}^{-1}
$$

where $c_{s}^{2}$ is the speed of sound squared of the matter source. We can expand this field in Fourier modes and canonically quantize it, introducing mode creation and annihilation operators $a_{k}^{\dagger}$ and $a_{k}$, respectively. 
The Hamiltonian derived from the quadratic action in momentum space is organized as follows (see e.g. [16])

$$
\begin{aligned}
H_{2}= & \frac{1}{2} \int \frac{d^{3} k}{(2 \pi)^{3}}\left[c_{s} k\left(a_{\boldsymbol{k}} a_{\boldsymbol{k}}^{\dagger}+a_{-\boldsymbol{k}} a_{-\boldsymbol{k}}^{\dagger}\right)\right] \\
& -\frac{1}{2} \int \frac{d^{3} k}{(2 \pi)^{3}}\left[i\left(\frac{z^{\prime}}{z}\right)\left(a_{\boldsymbol{k}} a_{-\boldsymbol{k}}-a_{\boldsymbol{k}}^{\dagger} a_{-\boldsymbol{k}}^{\dagger}\right)\right]
\end{aligned}
$$

where a prime denotes a derivative with respect to conformal time. The second term is the source of the two-mode squeezing term while the first one is the usual free part of the quadratic Hamiltonian, as in Minkowski space.

In the Heisenberg picture, the equation for $a_{k}$ is written

$$
\frac{d a_{\boldsymbol{k}}}{d \tau}=\frac{z^{\prime}}{z} a_{\boldsymbol{k}}^{\dagger}-i c_{s} k a_{\boldsymbol{k}}
$$

In the case of inflation $\frac{z^{\prime}}{z}=-\frac{1}{\tau}$. Hence, on super-Hubble scales (for which the momentum term on the right hand side of (15) is negligible), the $\mathrm{z}$ term results in a highly squeezed vacuum. In contrast, during a phase of slow Ekpyrotic contraction $\frac{z^{\prime}}{z}=-\frac{p}{(1-p) \tau}$ for super-Hubble modes. Since $p \ll 1$ the amount of squeezing is very small. In the limit $p \rightarrow 0$, the equation for the annihilation operators becomes the same as in flat spacetime quantum field theory.

Since the squeezing term is sub-dominant for Ekpyrosis, we can safely ignore the squeezing entropy which plays a crucial role for inflation, and focus on the entanglement entropy generated by the leading gravitational nonlinearities, i.e. by the cubic interaction term in the Hamitonian. This will be the topic of the following sections.

\section{ENTANGLEMENT ENTROPY FOR SCALAR PERTURBATIONS DUE TO GRAVITATIONAL NON-LINEARITIES}

In this section we turn to the computation of the momentum space entanglement entropy between super- and sub-Hubble modes generated by the gravitational nonlinearities. In applications to black hole physics and in the context of the AdS/CFT correspondence, entanglement entropy is usually considered in terms of position space entanglement [17. However, when considering the entropy of cosmological perturbations, it is more natural to consider momentum space entanglement. The reason is that the basic variables are the momentum modes of the fluctuations. The modes that classicalize and become accessible to cosmological experiments are the super-Hubble modes, with the sub-Hubble modes acting as a sea which are not directly accessible. Hence, it is natural to consider the entanglement between super- and sub-Hubble modes. In a previous paper [1, momentum space entanglement entropy was studied in the context of inflation, generalizing the formalism developed in 18 (see also [19, 20] for earlier work on the entropy of cosmological perturbations).

The entanglement entropy due to the mode coupling by the leading order cubic nonlinear terms can be calculated as follows. First, we break up the Hilbert space of scalar perturbations into sub-Hubble and super-Hubble modes, i.e.

$$
\mathcal{H}=\mathcal{H}_{\text {sub }} \otimes \mathcal{H}_{\text {super }} .
$$

The super-Hubble modes shall be treated as our system which is coupled to the bath of sub-Hubble modes. We shall carry out this calculation in Fourier space. Since our analysis is in the framework of an effective field theory, we need to apply $M_{\mathrm{Pl}}$ as a physical cutoff for our subHubble modes. The total Hamiltonian can be described as

$$
\mathbf{H}=\mathbf{H}_{\text {sub }} \otimes \mathbb{I}+\mathbb{I} \otimes \mathbf{H}_{\text {super }}+\mathbf{H}_{\text {int }},
$$

where $\mathbf{H}_{\text {sub }}$ and $\mathbf{H}_{\text {super }}$ refer to the quadratic Hamiltonian for the scalar modes with momenta $k<a H$ and $k>a H$, respectively. $\mathbf{H}_{\text {int }}$ refers to the interaction Hamiltonian due to our cubic non-Gaussian term.

As discussed in [1] (based on [21 and [22]), in terms of the variable $\zeta$, the integrated interaction Lagrangian is given by

$$
\begin{aligned}
& S_{3}=M_{\mathrm{Pl}}^{2} \int \mathrm{d} t \mathrm{~d}^{3} x {\left[a^{3} \epsilon^{2} \zeta \dot{\zeta}^{2}+a \epsilon^{2} \zeta(\partial \zeta)^{2}\right.} \\
&\left.-\frac{\mathrm{d}}{\mathrm{d} t}\left(a^{3} \epsilon^{2} \zeta^{2} \dot{\zeta}\right)\right]
\end{aligned}
$$

where we neglected nonlocal terms and used the fact that $\epsilon$ is constant. Since in the case of the Ekpyrotic scenario the dominant mode of $\zeta$ is constant, the leading interaction term in the action is

$$
S_{3}=M_{\mathrm{P} 1}^{2} \int \mathrm{d} t \mathrm{~d}^{3} x a \epsilon^{2} \zeta(\partial \zeta)^{2} .
$$

Using conformal time, and in terms of the canonical variable $v$, this interaction term takes the form

$$
S_{3}=\frac{\sqrt{\epsilon}}{2 \sqrt{2} a M_{\mathrm{Pl}}} \int \mathrm{d} \tau \mathrm{d}^{3} \mathbf{x} v(\partial v)^{2}
$$

To make contact with the formalism to compute the entanglement entropy developed in [18] and applied in [1] to the case of inflationary cosmology, we note that the effective coupling $\lambda$ is

$$
\lambda=\frac{\sqrt{\epsilon}}{2 \sqrt{2} a M_{\mathrm{Pl}}},
$$

from which we can define a dimensionless interaction parameter, given by

$$
\tilde{\lambda}=\frac{\sqrt{\epsilon} \Lambda}{2 \sqrt{2} a M_{\mathrm{Pl}}},
$$

where $\Lambda$ is a renormalization scale which we expect to be the Planck scale. 


\section{ENTANGLEMENT ENTROPY OF SCALAR PERTURBATIONS}

As a consequence of the nonlinearities, an initial vacuum state of both system and bath modes gets excited. At time $t$ the state $|\Omega\rangle$ becomes

$$
\begin{aligned}
|\Omega\rangle(t)=|0,0\rangle & +\sum_{n \neq 0} A_{n}(t)|n, 0\rangle+\sum_{n \neq 0} B_{N}(t)|0, N\rangle \\
& +\sum_{n, N \neq 0} C_{n, N}(t)|n, N\rangle
\end{aligned}
$$

where $|n\rangle$ denotes an n-particle state of the system (in fact, a product state over all super-Hubble $k$ modes), and $|N\rangle$ is the corresponding state for the bath. At the initial time, the coefficients $A_{n}, B_{N}$ and $C_{n, N}$ all vanish, and they build up gradually over time due to the gravitational interactions.

As shown in [18] and generalized in [1] in the case of a time-dependent background, the induced entanglement entropy between the super- and sub-Hubble modes is induced by the interaction coefficients $C_{n, N}$ in the following way

$$
S_{\mathrm{ent}}=-\lambda^{2} \log \left(\tilde{\lambda}^{2}\right) \sum_{n, N \neq 0}\left|\tilde{C}_{n, N}\right|^{2}
$$

For an infinite set of modes labelled by a continuous index $k$, the summation becomes a momentum space integral. We use the dimensionless effective coupling as the argument of the logarithm to make this term well-defined. However, in the end, this term will not be too important for our purposes as we shall ignore the logarithm term to focus only on the rest of the factors to get an order of magnitude estimate for the bound on the energy scale of the bounce.

We follow the analyses of 1 in order to calculate the entanglement entropy in this case. For the contracting phase in Ekpyrosis, the squeezing of the state of fluctuations on super-Hubble scale is negligible and we can set $r_{k}(\eta) \sim 0$ for the calculation. Hence, the general formula (62) of [1] simplifies to yield the following expression for the induced entanglement entropy density per comoving volume (please see the Appendix for details)

$$
\begin{aligned}
s & =(2 \pi)^{3} \lambda^{2} \log \left(\tilde{\lambda}^{2}\right) \int_{k_{I}}^{a H} \mathrm{~d}^{3} p_{3} \int_{a H}^{a M_{\mathrm{P} 1}} \mathrm{~d}^{3} p_{2} \int_{a H}^{a M_{\mathrm{Pl}}} \mathrm{d}^{3} p_{1} \delta^{3}\left(p_{1}+p_{2}+p_{3}\right) \frac{1}{\left(p_{1}+p_{2}+p_{3}\right)^{2}}\left(\frac{p_{1} p_{2}}{p_{3}}\right) \\
& =-\lambda^{2} \log \left(\tilde{\lambda}^{2}\right) \int_{k_{I}}^{a H} \frac{\mathrm{d}^{3} p_{3}}{(2 \pi)^{3}} \int_{a H}^{a M_{p l}} \frac{\mathrm{d}^{3} p_{2}}{(2 \pi)^{3}}\left(\frac{p_{2} \sqrt{p_{2}^{2}+p_{3}^{2}+2 p_{2} p_{3} \cos \theta}}{p_{3}}\right) \frac{1}{\left(\sqrt{p_{2}^{2}+p_{3}^{2}+2 p_{2} p_{3} \cos \theta}+p_{2}+p_{3}\right)^{2}}
\end{aligned}
$$

Notice that the super-Hubble mode $p_{3}$ gets an infrared cut-off by $k_{I}$ while the sub-Hubble mode $p_{2}$ has a natural, physical UV-cutoff given by $a M_{p l}$. The meaning of the IR cut-off can be thought of as follows: If there is a phase prior to Ekpyrosis, then we shall only consider super-Hubble modes generated during Ekpyrosis for our calculations. However, note that unlike in the case of inflation, we are free to take the limit $k_{I} \rightarrow 0$ for our integral without encountering any divergences. Generally we take $p_{3} \ll p_{2}$ when (25) is evaluated. Thus, we obtain

$$
\begin{aligned}
s & \approx \frac{\lambda^{2} \log \left(\tilde{\lambda}^{2}\right)}{4 \pi^{4}} \int_{k_{I}}^{a H} \mathrm{~d} p_{3} p_{3}^{2} \int_{a H}^{a M_{\mathrm{Pl}}} \mathrm{d} p_{2} p_{2}^{2} \frac{1}{4 p_{3}} \\
& \approx \frac{\lambda^{2} \log \left(\tilde{\lambda}^{2}\right)}{48 \pi^{4}}\left[(a H)^{2}-k_{I}^{2}\right] \cdot\left[\left(a M_{\mathrm{Pl}}\right)^{3}-(a H)^{3}\right] \\
& \sim \frac{1}{192 \pi^{4} p} \log \left(\frac{1}{2 \sqrt{2} p a}\right) a^{3} M_{\mathrm{Pl}} H^{2}
\end{aligned}
$$

In the last line, we have replaced the slow-roll parameter $\epsilon$ by the small number $p$ and set $\Lambda=M_{\mathrm{Pl}}$. Dividing by the factor $a^{3}$ we obtain the the entropy density per physical volume element. We see that the entanglement entropy (per unit physical volume) grows logarithmically as a function of time in the contracting phase, ignoring the time-dependence of $H$.

If we interpret entanglement entropy as a contribution to the entropy which should obey the second law of thermodynamics, then we can obtain an upper bound on the energy scale of the bounce (or equivalently on the value of $H$ just before the bounce) by demanding that the entanglement entropy 26 be smaller than the thermal entropy density after the bounce which is calculated as follows

$$
\frac{s}{V_{0}}=\frac{4}{3}\left(\frac{30}{\pi^{2} g}\right)^{1 / 4} \rho^{1 / 4}=\frac{4}{3}\left(\frac{90}{\pi^{2} g}\right)^{1 / 4} H^{3 / 2} M_{p l}^{3 / 2}
$$

where $g$ is the number of effective spin degrees of freedom in the thermal bath after the bounce. From 27) and 26) we obtain the condition

$$
\left(\frac{H}{M_{\mathrm{Pl}}}\right)^{1 / 2}<256 \pi^{7 / 2}\left(\frac{90}{g}\right)^{1 / 4} p .
$$

This sets an upper bound on the string scale, the energy scale where the S-brane will occur. It is not a very stringent bound. Assuming that the S-brane arises at 
string energy density, then the above equation yields the corresponding bound on the string energy scale in Planck units.

\section{CONCLUSIONS}

We have computed the entanglement entropy between sub- and super-Hubble modes during a phase of Ekpyrotic contraction. We found that this entropy grows logarithmically with decreasing scale factor. Demanding that the entanglement entropy at the end of the phase of contraction does not exceed the thermal entropy after the bounce, we obtain an upper bound on the energy scale of the bounce.

\section{Acknowledgments}

The research at McGill is supported in part by funds from NSERC and from the Canada Research Chair pro- gram. SB is supported in part by a McGill Space Institute fellowship and by a CITA National fellowship. ZW is supported in part by funds from a Templeton foundation sub-contract.

\section{Appendix: Calculation of entanglement entropy}

In order to explicitly calculate the entanglement entropy, we first need to evaluate the matrix elements, given by

$$
C_{n, N}:=-i \int_{\tau_{0}}^{\tau} \mathrm{d} \tau^{\prime} e^{i\left(p_{1}+p_{2}+p_{3}\right) \tau^{\prime}}\left\langle n, N\left|H_{\text {int }}\left(\tau^{\prime}\right)\right| 0,0\right\rangle(
$$

Note that the interaction Hamiltonian can be derived from 20 and is given by

$$
\begin{gathered}
\lambda(\tau) H_{\mathrm{int}}=\lambda(\tau) \int_{\Delta}\left[\sqrt{\frac{k_{2} k_{3}}{k_{1}}}\left(c_{-\mathbf{k}_{1}}^{\dagger} c_{-\mathbf{k}_{2}}^{\dagger} c_{-\mathbf{k}_{3}}^{\dagger}+c_{\mathbf{k}_{1}} c_{-\mathbf{k}_{2}}^{\dagger} c_{-\mathbf{k}_{3}}^{\dagger}+\ldots\right)+\sqrt{\frac{k_{2} k_{1}}{k_{3}}}\left(c_{-\mathbf{k}_{1}}^{\dagger} c_{-\mathbf{k}_{2}}^{\dagger} c_{-\mathbf{k}_{3}}^{\dagger}+\ldots\right)\right. \\
\left.+\sqrt{\frac{k_{1} k_{3}}{k_{2}}}\left(c_{-\mathbf{k}_{1}}^{\dagger} c_{-\mathbf{k}_{2}}^{\dagger} c_{-\mathbf{k}_{3}}^{\dagger}+\ldots\right)\right]
\end{gathered}
$$

where $\lambda$ is given by 21) and we have defined $\int_{\Delta}:=$ $\int \frac{\mathrm{d}^{3} k_{1}}{(2 \pi)^{3}} \frac{\mathrm{d}^{3} k_{2}}{(2 \pi)^{3}} \frac{\mathrm{d}^{3} k_{3}}{(2 \pi)^{3}}(2 \pi)^{3} \delta^{3}\left(\mathbf{k}_{1}+\mathbf{k}_{2}+\mathbf{k}_{3}\right)$ and the 'dots' are permutations.

Since the squeezing is negligible for Ekpyrosis, both the sub- and super-Hubble modes are in the Minkowski vacuum. This makes the evaluation of $\left\langle n, N\left|H_{\text {int }}\left(\tau^{\prime}\right)\right| 0,0\right\rangle$ relatively simpler compared to the case for inflation, and we need to only consider terms in $H_{\text {int }}$ of the form $c_{-\mathbf{k}}^{\dagger} c_{-\mathbf{k}}^{\dagger} c_{-\mathbf{k}}^{\dagger}$, where two of the modes can be sub-Hubble and one super-Hubble or the other way around. Once again, since the vacuum state is the same for both suband super-Hubble modes, the dominant contribution is going to be from the case where there are two super Hubble modes. We find:

$$
\left\langle n, N\left|H_{\text {int }}\left(\tau^{\prime}\right)\right| 0,0\right\rangle \sim \sqrt{\frac{p_{1} p_{2}}{p_{3}}},
$$

where $p_{1}, p_{2}$ are sub-Hubble and $p_{3}$ is the super-Hubble mode. (The other terms proportional to $\sqrt{p_{1} p_{3} / p_{2}}$ and $\sqrt{p_{2} p_{3} / p_{1}}$ are sub-dominant in this case.) Plugging this into the expression for the matrix element (29), we find

$$
C_{n, N}=-i \int_{\tau_{0}}^{\eta} \mathrm{d} \tau^{\prime} e^{i\left(p_{1}+p_{2}+p_{3}\right) \tau^{\prime}} \sqrt{\frac{p_{1} p_{2}}{p_{3}}}
$$

This is where the negligible squeezing for Ekpyrosis plays a crucial role so that we can evaluate the matrix elements as if for time-independent perturbation theory. Using this, one can calculate the entanglement entropy as 


$$
\begin{aligned}
& S_{\text {ent }} \sim(2 \pi)^{3} \int_{H}^{a H} \frac{\mathrm{d}^{3} p_{3}}{(2 \pi)^{3}} \int_{a H}^{a M_{\mathrm{P} 1}} \frac{\mathrm{d}^{3} p_{2}}{(2 \pi)^{3}} \int_{a H}^{a M_{\mathrm{P} 1}} \frac{\mathrm{d}^{3} p_{1}}{(2 \pi)^{3}} \delta^{3}\left(\mathbf{p}_{1}+\mathbf{p}_{2}+\mathbf{p}_{3}\right)\left(\frac{p_{1} p_{2}}{p_{3}}\right) \times \\
&\left|\int_{\tau_{0}}^{\tau} \mathrm{d} \tau^{\prime} e^{i\left(p_{1}+p_{2}+p_{3}\right) \eta^{\prime}}\right|^{2} \lambda^{2}\left(\tau^{\prime}\right) \log \left(\tilde{\lambda}^{2}\left(\tau^{\prime}\right)\right) .
\end{aligned}
$$

The time-dependence of the coupling parameter $\lambda(\tau)$ is extremely weak in Ekpyrosis since it is only due to the presence of the scale factor $a(\tau)$, which itself changes very slowly in conformal time. Therefore, one can pull the $\lambda^{2} \log \left(\tilde{\lambda}^{2}\right)$ term outside the integral and then one arrives at the result given in (24), with $\tilde{C}_{n, N}$ being the time-independent matrix elements. As shown in this Ap- pendix, in the case of Ekpyrosis, one can easily work with the time-independent matrix elements (due to the weak dependence of the interaction parameter on conformal time and having an usual flat vacuum for the superHubble modes) and we recover the results discussed in the main body of the text.
[1] S. Brahma, O. Alaryani and R. Brandenberger, "Entanglement Entropy of Cosmological Perturbations," Phys. Rev. D 102, no. 4, 043529 (2020) doi:10.1103/PhysRevD.102.043529 arXiv:2005.09688 [hep-th]].

[2] A. Bedroya, R. Brandenberger, M. Loverde and C. Vafa, "Trans-Planckian Censorship and Inflationary Cosmology," Phys. Rev. D 101, no. 10, 103502 (2020) doi:10.1103/PhysRevD.101.103502 arXiv:1909.11106 [hep-th]].

[3] A. Bedroya and C. Vafa, "Trans-Planckian Censorship and the Swampland," arXiv:1909.11063 [hep-th].

[4] R. Brandenberger and Z. Wang, "Nonsingular Ekpyrotic Cosmology with a Nearly Scale-Invariant Spectrum of Cosmological Perturbations and Gravitational Waves," Phys. Rev. D 101, no. 6, 063522 (2020) doi:10.1103/PhysRevD.101.063522 arXiv:2001.00638 [hep-th]].

[5] J. Khoury, B. A. Ovrut, P. J. Steinhardt and N. Turok, "The Ekpyrotic universe: Colliding branes and the origin of the hot big bang," Phys. Rev. D 64, 123522 (2001) hep-th/0103239;

J. Khoury, B. A. Ovrut, N. Seiberg, P. J. Steinhardt and N. Turok, "From big crunch to big bang," Phys. Rev. D 65, 086007 (2002) doi:10.1103/PhysRevD.65.086007 hep-th/0108187.

[6] D. Baumann and L. McAllister, "Inflation and String Theory," doi:10.1017/CBO9781316105733 arXiv:1404.2601 [hep-th].

[7] J. K. Erickson, D. H. Wesley, P. J. Steinhardt and N. Turok, "Kasner and mixmaster behavior in universes with equation of state $w i=1$," Phys. Rev. D 69, 063514 (2004) doi:10.1103/PhysRevD.69.063514 hep-th/0312009.

[8] W. G. Cook, I. A. Glushchenko, A. Ijjas, F. Pretorius and P. J. Steinhardt, "Supersmoothing through Slow Contraction," arXiv:2006.01172 [gr-qc].

[9] R. Brandenberger and P. Peter, "Bouncing Cosmologies: Progress and Problems," Found. Phys. 47, no. 6, 797 (2017) doi:10.1007/s10701-016-0057-0 arXiv:1603.05834 [hep-th]].
[10] M. Gutperle and A. Strominger, "Space - like branes," JHEP 0204, 018 (2002) doi:10.1088/1126$6708 / 2002 / 04 / 018$ hep-th/0202210.

[11] R. Brandenberger and Z. Wang, "Ekpyrotic Cosmology with a Zero-Shear S-Brane," Phys. Rev. D 102, no. 2, 023516 (2020) doi:10.1103/PhysRevD.102.023516 arXiv:2004.06437 [hep-th]].

[12] R. Brandenberger, K. Dasgupta and Z. Wang, "Reheating after S-Brane Ekpyrosis," arXiv:2007.01203 [hep-th].

[13] V.F. Mukhanov, H.A. Feldman and R.H. Brandenberger, "Theory of Cosmological Perturbations" Physics Reports 215, 203 (1992).

[14] R. H. Brandenberger, "Lectures on the theory of cosmological perturbations," Lect. Notes Phys. 646, 127 (2004) doi:10.1007/978-3-540-40918-25 hep-th/0306071.

[15] C. Kiefer, D. Polarski and A. A. Starobinsky, "Quantum to classical transition for fluctuations in the early universe," Int. J. Mod. Phys. D 7, 455 (1998) grqc/9802003.

[16] E. Nelson, "Quantum Decoherence During Inflation from Gravitational Nonlinearities," JCAP 1603, 022 (2016) arXiv:1601.03734 [gr-qc]].

[17] P. Calabrese and J. L. Cardy, "Entanglement entropy and quantum field theory," J. Stat. Mech. 0406, P06002 (2004) hep-th/0405152;

J. Eisert, M. Cramer and M. B. Plenio, "Area laws for the entanglement entropy - a review," Rev. Mod. Phys. 82, 277 (2010) arXiv:0808.3773 [quant-ph]];

S. Ryu and T. Takayanagi, "Holographic derivation of entanglement entropy from AdS/CFT," Phys. Rev. Lett. 96, 181602 (2006) hep-th/0603001;

S. Ryu and T. Takayanagi, "Aspects of Holographic Entanglement Entropy," JHEP 0608, 045 (2006) hepth/0605073;

T. Nishioka, S. Ryu and T. Takayanagi, "Holographic Entanglement Entropy: An Overview," J. Phys. A 42, 504008 (2009) arXiv:0905.0932 [hep-th]].

[18] V. Balasubramanian, M. B. McDermott and M. Van Raamsdonk, "Momentum-space entanglement and renormalization in quantum field theory," Phys. Rev. D 86, 045014 (2012) arXiv:1108.3568 [hep-th]]. 
[19] S. S. Kumar and S. Shankaranarayanan, "Role of spatial higher order derivatives in momentum space entanglement," Phys. Rev. D 95 (2017) no.6, 065023 arXiv:1702.08655 [hep-th]].

[20] R. H. Brandenberger, V. F. Mukhanov and T. Prokopec, "Entropy of a classical stochastic field and cosmological perturbations," Phys. Rev. Lett. 69, 3606 (1992) astroph/9206005;

R. H. Brandenberger, T. Prokopec and V. F. Mukhanov, "The Entropy of the gravitational field," Phys. Rev. D 48, 2443 (1993) gr-qc/9208009;

M. Gasperini and M. Giovannini, "Entropy production in the cosmological amplification of the vacuum fluctua- tions," Phys. Lett. B 301, 334 (1993) gr-qc/9301010; M. Gasperini and M. Giovannini, "Quantum squeezing and cosmological entropy production," Class. Quant. Grav. 10, L133 (1993) gr-qc/9307024.

[21] P. Adshead, W. Hu, C. Dvorkin and H. V. Peiris, "Fast Computation of Bispectrum Features with Generalized Slow Roll," Phys. Rev. D 84, 043519 (2011) arXiv:1102.3435 [astro-ph.CO]].

[22] J. M. Maldacena, "Non-Gaussian features of primordial fluctuations in single field inflationary models," JHEP 0305, 013 (2003) astro-ph/0210603. 